\title{
Social psychological characteristics and regional cultural identity of male drug addicts: A Case Study of Southern shandong
}

\author{
Hairong Zhu, a, Jinpeng Yan ${ }^{2, b}$ \\ ${ }^{1}$ School of Psychology and Education Science, Zaozhuang University, Zaozhuang, Shandong, \\ China \\ ${ }^{2}$ Zaozhuang compulsory isolation drug rehabilitation center, Zaozhuang, Shandong, China \\ ${ }^{3}$ List all distinct addresses in the same way \\ a54756783@qq.com, b505284368@qq.com
}

\begin{abstract}
Keywords: Regional culture, male drug addicts, personality traits, Sworn brothers.
Abstract. In this study, 64 cases of forced isolation drug addicts conducted a general survey, a number of Minnesota Personality Test (MMPI). The results showed that male drug addicts in Southern shandong region generally had low cultural level;Family economic situation was in general;Divorce rate was higher; Sworn brother phenomenon was common than in general population; Southern shandong drug addicts' personality was not beyond normal range, but compared with general population, there was obvious personality deviation. Male drug addicts in Southern shandong region have common characteristics with other drug addicts, and also show obvious regional cultural characteristics.
\end{abstract}

\section{男性吸毒者的社会心理特征与区域文化认同 一以鲁南地区为例 \\ 朱海荣 $1, a$, 晏金鹏 $2, b,{ }^{*}$ \\ 1本庄学院心理与教育科学学院, 苯庄, 山东, 中国 2本庄市强制隔离戒毒所, 菄庄, 山东, 中国 \\ a54756783@qq.com, b505284368@qq.com}

关键词：区域文化;男性吸毒者;人格特征; 结拜兄弟

中文摘要. 本研究对64名强制隔离戒毒人员进行了一般情况调查、明尼苏达多项人格测试

(MMPI), 结果显示: 鲁南地区男性吸毒者文化水平普遍较低, 家庭经济状况一般, 离婚率 较高; 结拜仁兄弟现象较一般人群普遍, 吸毒与其有明显关系; 鲁南地区性吸毒者人格并未 超出正常范围, 但与普通群体相比, 存在明显的人格偏差。尤其在诈病、疑病、精神病态、 妄想、精神衰弱、精神分裂和轻躁狂几个方面表现出明显的人格偏差。鲁南地区男性吸毒者 与其他吸毒者既有社会及心理特征的共性，又显现出明显的区域文化特点。

\section{1. 引言}

我国吸毒人群已突破以往高发于边境、沿海地区的地域特征，遍及全国各省份。鲁南地 区是山东的南大门, 沟通南北, 交通便利, 与山东沿海地区相比, 其工业化、城市化水平不 高, 经济外向度偏低。其地域、经济、文化等特点决定了鲁南地区吸毒群体的特点, 戒毒工 作要着眼于区域经济、文化、民俗特点，有针对性地开展心理、社会康复工作，以达到更好 的戒毒效果。 


\section{2.研究方法}

\section{1被试}

本研究对象选自柊庄市强制隔离戒毒所内近期入所的68名男性吸毒者, 年龄在21-45岁之 间。

\section{2测量工具}

(1) 一般资料调查问卷 采用自编一般资料调查问卷对束庄市强戒所68名男性吸毒者进 行基本资料的调查，包括个人基本资料，家庭状况及与吸毒相关的情况。

（2）明尼苏达多项人格测验（简称MMPI）采用该问卷对吸毒者的人格特征进行测验。 本测验共 566 题, 实际为 550 题, 其中 16 题为重复题。共包含 13 个量表, 其中 10 个临床量表 (Hs 疑病、D抑郁、Hy癔病、Pd精神病态、Mf男子气-女子气、Pa妄想狂、Pt精神衰弱、Sc精神分 裂症、Ma轻躁狂、Si社会内向), 3 个效度量表（L说谎、F诈病、K校正）。

\section{3数据处理}

(1) 采用 spss17.0对数据进行统计分析。

(2) 为保证问卷的有效性, 将答卷不完整, $\mathrm{L}$ 分在 10 分以上或者 $\mathrm{Q}$ 分在 25 分以上的答卷进 行剔除，最后选出有效问卷64份。

\section{3. 结果}

\section{1人口学资料情况}

\section{1.1 个人资料}

调查显示, 64名男性吸毒者平均年龄在 27 岁，其中最大的 45 岁，最小的 21 岁。

表1. 鲁南男性吸毒者个人资料

\begin{tabular}{|c|c|c|c|c|c|c|c|c|c|}
\hline & \multicolumn{3}{|c|}{ 婚姻状况 } & \multicolumn{4}{|c|}{ 文化程度 } & \multicolumn{2}{|c|}{ 是否有子女 } \\
\hline & 已婚 & 未婚 & 离婚 & 大学 & $\begin{array}{l}\text { 高中/ } \\
\text { 中专 }\end{array}$ & 初中 & $\begin{array}{c}\text { 小学 } \\
\text { 及以 } \\
\text { 下 }\end{array}$ & 是 & 否 \\
\hline 人数 & 23 & 25 & 26 & 0 & 13 & 37 & 14 & 39 & 25 \\
\hline 比例 & $35.9 \%$ & $39.1 \%$ & $25.0 \%$ & $0 \%$ & $20.1 \%$ & $57.8 \%$ & $21.9 \%$ & $60.9 \%$ & $39.1 \%$ \\
\hline
\end{tabular}

从表 1 可知, 本次调查的吸毒者中未婚人数和已婚人数差距不大, 但值得注意的是离婚率 占了总人数的四分之一, 比例较大; 文化程度大都在初中水平, 高中或小学以下也占有一定 比例, 没有大学生, 文化水平普遍较低; 有子女的人数较多。

3.1.2家庭情况

从表 2 可知, 本次调查的吸毒者大部分生在完整的家庭, 家庭氛围比较融洽或一般, 极少 数家庭关系紧张, 大部分为非独生子女, 父母去世或离婚的人数占总调查人数的四分之一, 比例较高；大部分家庭收入处于中低水平；职业大都为商人，农民和其他。

表2. 鲁南男性吸毒者家庭情况调查表

\begin{tabular}{|c|c|c|c|}
\hline & & 人数 & 比例 \\
\hline \multirow{3}{*}{$\begin{array}{c}\text { 家庭基本情 } \\
\text { 况 }\end{array}$} & 家庭完整 & 47 & $73.4 \%$ \\
\cline { 2 - 4 } & $\begin{array}{c}\text { 父母方去世或 } \\
\text { 离婚 }\end{array}$ & 16 & $25.0 \%$ \\
\cline { 2 - 4 } & 双方去世 & 1 & \\
\hline \multirow{3}{*}{ 个人月收入 } & $1000-3000$ & 30 & $4.6 \%$ \\
\cline { 2 - 4 } & $3500-5000$ & 28 & $43.8 \%$ \\
\cline { 2 - 4 } & $5500-8000$ & 4 & $6.3 \%$ \\
\cline { 2 - 4 } & 8000 以上 & 2 & $3.1 \%$ \\
\hline 是否为独生 & 是 & 11 & $82.8 \%$ \\
\cline { 2 - 4 } 子女 & 否 & 53 & $59.4 \%$ \\
\hline 原生家庭氛 & 融洽 & 38 & \\
\hline
\end{tabular}




\subsection{3吸毒情况}

\begin{tabular}{|c|c|c|c|}
\cline { 2 - 4 } \multicolumn{1}{|c|}{} & 一般 & 23 & $35.9 \%$ \\
\cline { 2 - 4 } & 紧张 & 3 & $4.7 \%$ \\
\hline 家庭成员是 & 是 & 1 & $1.6 \%$ \\
\hline 否有吸毒的 & 否 & 63 & $98.4 \%$ \\
\hline \multirow{4}{*}{} & 工人 & 3 & $4.7 \%$ \\
\cline { 2 - 4 } & 农民 & 18 & $28.1 \%$ \\
\cline { 2 - 4 } & 公司职员 & 2 & $3.1 \%$ \\
\cline { 2 - 4 } & 经商 & 22 & $34.4 \%$ \\
\cline { 2 - 4 } & 干部、教师、医 & 1 & $1.6 \%$ \\
\cline { 2 - 4 } & 生 & & \\
\cline { 2 - 4 } & 待业 & 3 & $0.7 \%$ \\
\cline { 2 - 4 } & 学生 & 0 & $23.4 \%$ \\
\cline { 2 - 4 } & 其他 & 15 & \\
\hline
\end{tabular}

毒品种类：全部为冰毒。吸食年限：最多13年，最少半年，平均吸食3.5年。在调查的 64 人中复吸人数为 38 人, 所占比例为 $59.38 \%$ 。

表3. 鲁南男性吸毒者吸毒原因调查表

\begin{tabular}{|c|c|c|c|}
\hline \multirow{4}{*}{ 吸食原因 } & 原因 & 人数 & 比例 \\
\hline & 好奇 & 50 & $78.1 \%$ \\
\cline { 2 - 4 } & 同伴影响 & 45 & $70.3 \%$ \\
\cline { 2 - 4 } & 生病止痛 & 0 & $0 \%$ \\
\cline { 2 - 4 } & 心情苦闷 & 19 & $29.7 \%$ \\
\cline { 2 - 4 } & 其他 & 11 & $17.2 \%$ \\
\hline 复吸原因 & 解除心情烦问 & 17 & $44.7 \%$ \\
\cline { 2 - 4 } & 无聊 & 9 & $23.7 \%$ \\
\cline { 2 - 4 } & 以前毒友引诱 & 16 & $42.1 \%$ \\
\cline { 2 - 4 } & 社会压力 & 9 & $23.7 \%$ \\
\cline { 2 - 4 } & 戒断症状 & 3 & $7.9 \%$ \\
\cline { 2 - 4 } & 回到旧环境 & 25 & $65.9 \%$ \\
\cline { 2 - 4 } & 再吸最后一次 & 5 & $13.2 \%$ \\
\cline { 2 - 4 } & 其他 & 3 & $7.9 \%$ \\
\hline
\end{tabular}

从表 3 可知, 本次调查的吸毒者的吸毒原因大部分是由于好奇, 和受同伴影响, 没有为了 生病止痛的, 还有一部分原因为心情苦闷和其他; 复吸的主要原因是解除心情烦问和过去毒 友引诱, 还有一部分原因是打发时间, 社会压力和回到旧环境。

3.1.4区域文化认同特点

调查显示, 64 名被试中有 60 名有拜仁兄弟行为, 比例高达 $97.8 \%$, 并表示其吸毒与复吸与 仁兄弟朋友圈有密切关系，呈现出较独特的区域文化特点。

\section{2 人格测试结果}

将 64例男性吸毒者 MM PI测查原始分与全国常模各量表原始分调查结果进行比较，结 果见表 4 。

如表4所示, 男性吸毒者在 13 个分量表上, 均与全国常模存在非常显著的差异 (P $<0.001)$ 。其中，F、Hs、Pd、Pa、Pt、Sc、Ma 7个量表得分显著高于全国常模，这表明男性 吸毒者和正常人有很大差别, 或者说存在明显的人格偏向, 这种人格偏差具有一致性和整体 性, 平均T分均没有超过60分, 说明男性吸毒者在诈病、疑病、精神病态、妄想、精神衰弱、 精神分裂和轻躁狂几个方面表现出明显的人格偏差, 但没有达到异常水平。具体分析如下:

（1）在L量表上，男性吸毒者的得分低于常模，这表明吸毒者愿意承认他们的缺点和不 
足之处, 这与他们所处的环境有关, 表现好的可以得到表扬, 这关系到他们能否顺利出所。 因此，他们会尽量利用一些场合表现其诚实。

（2）与普通群体相比, 吸毒者 $\mathrm{F}$ 量表的分数要高, 说明受试者不认真, 或理解错误, 表 现出一种互相无关的症状或伪装疾病。

（3）在 $K$ 量表上, 吸毒者得分低于正常人, 这与 $L$ 量表和 $F$ 量表相对应, 表明吸毒者对自 己有不满或自我批判, 表现出对他人的怀疑和不信任, 因此, 要走进他们的内心世界首先要 与他们建立良好的信任关系。

（4）此次调查中，吸毒者的D、Hy、Mf、Si量表得分均低于全国常模，表明了吸毒者的 精神状态良好，没有癔病和抑郁症状，人格偏向于外向、爱社交、富于表情、易激惹、健谈 且不受拘束。

（5）在Hs量表上，吸毒者表现为许多身体上的不适，不愉快，自我中心，敌意需求，博 同情, 这也与他们所处的环境有关, 与外界很少交流, 被强制戒毒, 生活单调, 还有部分被 试停止吸毒之后出现的一系列身心戒断症状。

（6）在Pd、Pa、Pt、Ma、Sc的分量表得分来看，吸毒者表现出：行为上表现出敌意、多 疑、好争论、无视道德的约束，不信任他人也不被他人信任，具有攻击倾向；过分敏感、紧 张、孤独、烦恼, 经常自罪自责, 心情阴晴多变, 反复思考, 强迫思想; 善交际、精力旺盛、 绝对乐观、过分高估自己、爱享受、虚伪做作、好出风头, 表现力和判断力差; 有时候他们 缺乏对社会的归属感, 常常自卑, 感到被孤立、被疏远, 感到广泛的焦虑, 也表现出性角色 的混乱和过分的关心。

表4. 鲁南男性吸毒者各分量表原始分和T分均值与全国常模的比较 $(X \pm S)$

\begin{tabular}{|c|c|c|c|c|}
\hline 项目 & $\begin{array}{c}\text { 男性吸毒者 } \\
\mathrm{n}=64\end{array}$ & $\begin{array}{c}\text { 全国男性常模 } \\
\mathrm{N}=1553\end{array}$ & $\mathrm{t}$ 值 & $\begin{array}{c}\text { 男性吸毒者 } \\
\text { 平均分 }\end{array}$ \\
\hline $\mathrm{L}$ & $3.80 \pm \mathrm{s} 1.83$ & $5.70 \pm \mathrm{s} 2.52$ & $15.964 * * *$ & 42.19 \\
\hline $\mathrm{F}$ & $14.88 \pm \mathrm{s} 5.14$ & $13.68 \pm \mathrm{s} 6.86$ & $22.225 * * *$ & 51.34 \\
\hline $\mathrm{K}$ & $10.12 \pm \mathrm{s} 4.11$ & $13.00 \pm \mathrm{s} 4.66$ & $18.926 * * *$ & 43.32 \\
\hline $\mathrm{Hs}$ & $8.92 \pm \mathrm{s} 4.54$ & $8.78 \pm \mathrm{s} 4.75$ & $15.077 * * *$ & 47.25 \\
\hline $\mathrm{D}$ & $25.25 \pm \mathrm{s} 4.59$ & $26.16 \pm \mathrm{s} 4.97$ & $42.240 * * *$ & 47.51 \\
\hline $\mathrm{Hy}$ & $20.17 \pm \mathrm{s} 5.13$ & $22.07 \pm \mathrm{s} 5.36$ & $30.181 * * *$ & 46.02 \\
\hline $\mathrm{Pd}$ & $23.00 \pm \mathrm{s} 4.79$ & $18.98 \pm \mathrm{s} 4.36$ & $36.893 * * *$ & 56.17 \\
\hline $\mathrm{Mf}$ & $26.64 \pm \mathrm{s} 3.66$ & $27.56 \pm \mathrm{s} 4.04$ & $55.968 * * *$ & 47.27 \\
\hline $\mathrm{Pa}$ & $14.05 \pm \mathrm{s} 4.08$ & $12.84 \pm \mathrm{s} 3.92$ & $26.461 * * *$ & 52.49 \\
\hline $\mathrm{Pt}$ & $23.76 \pm \mathrm{s} 8.20$ & $17.86 \pm \mathrm{s} 7.93$ & $22.268 * * *$ & 55.76 \\
\hline $\mathrm{Sc}$ & $27.88 \pm \mathrm{s} 10.26$ & $23.01 \pm \mathrm{s} 10.15$ & $20.879 * * *$ & 51.90 \\
\hline $\mathrm{Ma}$ & $20.71 \pm \mathrm{s} 4.56$ & $18.48 \pm \mathrm{s} 5.26$ & $34.916 * * *$ & 53.03 \\
\hline $\mathrm{Si}$ & $32.00 \pm \mathrm{s} 7.72$ & $34.51 \pm \mathrm{s} 6.88$ & $31.842 * * *$ & 45.92 \\
\hline
\end{tabular}

***表示p $\mathrm{p}<0.001$

\section{4. 讨论}

\section{1男性吸毒者人口学特点}

鲁南地区男性吸毒者普遍年龄偏小, 文化程度不高, 大部分人只读到初中阶段, 最高文 化程度为高中, 一部分人的文化程度为小学及以下, 对毒品的危害认知贫乏甚至错误, 因此 沾染毒品的几率比较大; 从经济状况来看: 吸毒者人均月收入普遍偏低, 且以经商和农民为职 业者居多, 由于文化素质不高, 吸毒者所从事的工作较为单一, 从而表现出对工作、对生活 的不满, 在社会中的自尊感、自我价值感偏低, 毒品成为其产生自尊感获得感的替代品; 家 庭方面, 吸毒者多半为非独生子女, 原生家庭的完整性及家庭氛围融洽者占大多数, 但吸毒 
者离婚率占所有被试的四分之一，夫妻关系一般或紧张者占总人数的四成，比例相对较高， 综上分析, 大部分吸毒者在原生家庭中并不缺少父母之爱, 但父母的家庭教养方式存在问题, , 吸毒者成长过程中缺乏正确的管教和引导, 吸毒者缺乏维系长期亲密情感的能力。因此家庭 因素是致使吸毒的因素之一。

\section{2男性吸毒者人格特征分析}

虽然人格没有好坏之分，但不同人格特点会影响个体的行为方式和对环境的应对方式。 MMPI测试结果表明, 鲁南地区男性吸毒者在 13 个分量表上，均与全国常模存在非常显著的差 异。其中, F、Hs、Pd、Pa、Pt、Sc、Ma7个量表得分显著高于全国常模, 说明在计病、疑病、 精神病态、妄想、精神衰弱、精神分裂和轻躁狂几个方面, 吸毒者表现出明显的人格偏差, 但并未达到异常水平, 这是值得重视的特点, 说明男性吸毒者与普通人群相比, 存在某种程 度上的人格缺陷, 例如: 依赖性强、过分焦虑紧张、缺乏社会责任感和自制力, 缺乏对他人、 社会的信任, 与社会的亲和力低, 社会价值感不高, 他们往往难以应付生活挑战, 吸毒者受 制于外部毒品控制的力量很强, 对自己行为的内控力不足。吸毒者的人格倾向及心理特征, 导致其更容易借用毒品来麻醉感觉，逃避现实。

\section{3社会心理特征与区域文化认同的关系}

结拜兄弟这种亚文化现象，在中国下层社会中，有着悠久的历史渊源和广大的群众根基。 鲁南地区的束庄煤矿开发历史悠久, 㫫庄煤矿在全国煤炭工业史上占有重要位置。㫫庄矿山 文化的形成和发展，与鲁南地区传统民风交织在一起，营造了矿山特有的生存环境。人与人 之间的交往，超越了血缘、亲缘和地缘关系，鲁南地区又受儒家 “仁义” 思想的影响明显， 朋友以情义为基础, 因此, 结拜兄弟行为特别盛行, 直至今日, 菄庄虽已进行由资源型城市 向文化旅游城市转型, 结拜兄弟之风较其他地区依然显著, 并且延续了矿山文化中崇尚吃喝、 今日有酒今日醉的特点。调查中显示, 鲁南地区男性吸毒者中有结拜兄弟行为的高达 $97.75 \%$, 这种交往关系缺乏稳固的纽带, 他们维系关系的重要纽带之一就是在一起吃喝玩乐, 随着社 会的发展, 吸毒成为该群体的选择, 与结拜兄弟聚集, 受同伴诱惑, 认为大家都吸、身不由 己、顺应潮流的思想普遍存在, 因此其吸毒与复吸与仁兄弟朋友圈有密切关系。结义之风是 鲁南地区长久以来群体价值观念、生活方式、行为方式、民族习俗的内化与外显, 这种区域 文化认同咜存了人们的情感和人际联系, 为生活赋予意义和目的。根据马斯洛需要层次理论, 由于吸毒者自身社会生活状况普遍不佳, 普遍存在一定程度的人格缺陷, 社会价值感不高, 结拜兄弟满足了其归属的需要、尊重的需要, 吸毒又成为维系关系、满足虚假认同的载体。 如何看待与应对结拜兄弟现象，是使其彻底脱离毒品必须面对的问题。

\section{5.结论}

（1）鲁南地区男性吸毒者文化程度普遍偏低，婚姻关系欠佳，家庭责任感低，经济状况 一般或较差, 社会适应能力较差。

（2）男性吸毒者人格并未超出正常范围, 但与普通群体相比, 存在明显的人格缺陷。要 加强心理干预，改变反社会态度，改善人际关系模式，提高自我控制和自我管理的能力。

（3）男性吸毒者的社会心理特征呈现出独特的区域文化认同特点。鲁南地区男性吸毒者 结拜兄弟现象盛行, 不利于其彻底脱离毒品。中国不同地区区域文化有很大不同, 应将区域 文化因素纳入戒毒干预体系。

\section{致谢}

本文为東庄学院 “十三五”重点学科教育学（课程与教学论）的阶段性成果之一。 


\section{References}

[1] José Luis Carballo, Ainhoa Coloma-Carmona and Dana Mrozowicz-Gaudyn, Psychological assessment of opioid drug abuse, Papeles del Psicólogo, . Vol. 37(1), pp. 45-51,2016.

[2] Amin Gousheh, Hassan Ziaaddini and Mohammad Reza Baneshi, Drug Use among Residents of Juvenile Correctional Center in Kerman, Iran, and its Relationship with Personality Dimensions and Self-concept, Addiction \& Health, 6(1-2).pp.22-29,2014.

[3] Eyn Yuen, Jw Toumbourou ,Does family intervention for adolescent substance use impact parental mental health?A systematic review, Advances in Mental Health, 7(7).pp.186-199,2014.

[4] Wu Xiaoyan,Zeng Hong and Li Ling, Nevestigation and analysis of the motivativation for abstaining from drugs in 1294 drug addicts, Chin J Drug Depend, 18(3), pp.207-214, 2009.

[5] Liu chang, Personality characteristics and psychological correction of addicts under treatment, Chinese Journal of Clinical Rehabilitation, Vol.10 No.10, pp.155-157,2006

[6] Zhou Caichun,Deng Juping and Li Weiping, Relapse factors among drug abusers after voluntary drug-treatment and rehabilitation through labor, Chin J Drug Depend, 20(2), pp.131-135, 2011

[7] Hu xiaolin,Lunan regional culture,Hiongher education Press, Beijing, pp.170-178, 2013

[8] Wang mingyuan, An Anthropological Study of the "Brotherhood phenomenon " in Zaozhuang, Shandong Province, folklore studies, vol.107, 2013. 